\title{
Relapsing fever outbreak investigation in Bahir-Dar, Amhara Region, Ethiopia
}

\author{
Addisu Workineh Kassa ${ }^{1,3, *}$, Amelework Getnet Alene ${ }^{1,3}$, Tilahun Belete Mosie , $^{2}$ \\ ${ }^{1}$ Public Health Emergency Management, Bahir Dar, Ethiopia \\ ${ }^{2}$ Felegehiwot Referral Hospital, Bahir Dar, Ethiopia \\ ${ }^{3}$ Amhara regional Health Bureau, Bahir Dar, Ethiopia
}

\section{Email addresses:}

analid9800@gmail.com (A. W. Kassa),aminefse@yahoo.com (A. G. Alene), tilahunbe100@gmail.com (T. B. Mosie)

\section{To cite this article:}

Addisu Workineh Kassa, Amelework Getnet Alene, Tilahun Belete Mosie. Relapsing Fever Outbreak Investigation in Bahir-Dar, Amhara Region, Ethiopia. Science Journal of Public Health. Vol. 2, No. 4, 2014, pp. 284-287. doi: 10.11648/j.sjph.20140204.16

\begin{abstract}
Introduction: Ethiopia is the main endemic focus of louse borne relapsing fever. Borrelia recurrentis is the etiologic agent for louse-borne relapsing fever and occurs as epidemic under conditions of poor socio economic status, overcrowding, poverty, draught and famine. Methods: Unmatched community based case control and descriptive cross sectional investigation were conducted. All patients from 15 October-15 November 2012 were included. Sixty eight patients matched with 136 controls that had no previous history of relapsing fever living in the same village. Study was conducted in in households with individuals who go to work on a daily basis and data was analyzed using Microsoft Excel and Epi-Info version 7.1.0.6.Results: Among all confirmed patients 100\% (68), 92.6\% (63), 94.1\% (64) and 82.3\% (56) presented with fever, head ache, chills and vomiting respectively. There was no death during the outbreak. Mean age of respondents was 21 years and their median age was 20 years (SD 7.14). Statistically significant associations were observed between 51 (75\%) patients and 27.2\% (37) controls who did wash their clothes at least weekly (AOR = 13.23, 95\% CI [5.51-31.75]) and 79.4\% (54) patients and 64\% (87) controls who did not take body bath at least weakly (AOR = 8.01, 95\%CI [3.51-18.29]) and the associations were statistically significant. Those not washing clothes and taking baths at least weekly had a 13 and 8 fold likelihood of acquiring relapsing fever compared to those who wash clothes and take baths respectively at least on a weekly basis. Conclusion: Poor personal hygiene was contributed to the outbreak. The outbreak was contained due to prompt interventions taken and strong preventive measures were recommended to prevent emergence of future outbreak of relapsing fever.
\end{abstract}

Keywords: Borrelia recurrentis, Relapsing Fever, Bahir Dar, Ethiopia

\section{Introduction}

Relapsing fever (RF) was once a disease of global epidemic importance. Among twenty-seven borrelia species, fifteen are known to be associated with RF. Borrelia recurrentis is the only etiologic agent of louse-borne (LB) $\mathrm{RF}$ and humans are the only known reservoirs[1]. It is transmitted from human to human by the body louse, Pediculus humanus. The pathogen multiplies in the gut of the louse and is transmitted when an infected louse is crashed or scratched while feeding in the human host[2].

Louse-borne relapsing fever has been restricted to countries with poor socio economic status, the most important foci being Burundi, Rwanda and Ethiopia. Borrelia recurrentis is the etiologic agent for louse-borne relapsing fever and occurs as epidemic under conditions of overcrowding, poverty, draught and famine. Homeless people in crowded shelters are also at risk of louse born relapsing fever[3], [4].

Large outbreaks of louse-borne relapsing fever had occurred throughout the past century. These outbreaks usually occur following man-made break downs in public health, as typified by the epidemic following World War II that involved about 10 million cases and one million deaths during this epidemic[3].

Currently, epidemic relapsing fever is found only in Ethiopia and neighbouring countries, although its occurrence among homeless people of industrialized European cities has been suspected but not confirmed. Famine, war, and the movement and groups of refugees often result in epidemics of louse born relapsing fever[5], [6]. 
Ethiopia is main endemic focus of louse borne relapsing fever. It was reported as seventh of the top ten leading causes of admission and death among adults in the country, in 2002/03. More than 9000 cases were reported to the ministry of health in the same year. However, lack of diagnostic facilities in rural health set up and incomplete reporting make it difficult to estimate total number of cases [3], [7].

Several large epidemics were recorded in the country, usually following war and famine. Localized epidemics continue to occur when circumferences become favourable. The latest epidemic occurred in 1991/92, at the end of the civil war in Ethiopia. It occurred among military recruits returning to their residence areas, and later spread to different sections of the community, including schools. Among 389 patients from Arsi, southern Ethiopia, during this epidemic, the case fatality rate was $3.5 \%$ [3].

Infestation was more frequent in high lands where people bath and wash clothes less frequently, and uses more bedding. A more recent study of prevalence of lice infestation among school children showed that $66.8 \%$ of the students harboured body lice. The prevalence was significantly higher in Debre-Berhan, $76.4 \%$, at an altitude of 2850 meters, compared to Gambella, $60.3 \%$, located at an altitude of 485 meters above sea level. In urban areas, the disease occurs mainly among jobless migrants, daily labourers, prisoners and the poor[3].

The aim of investigation was to investigate the occurrence of relapsing fever, identify the risk factors and suggest practical control measures to alleviate the disease burden of the community in Bahir-Dar.

\section{Methodology}

\subsection{Study Area}

Bahir-Dar city administratively divided in to nine urban, three satellite towns and nine rural kebeles. There is one referral hospital, 10 health centers and 10 health posts; also the city has two private hospitals, 36 private and two NGO clinics. There were 20 rural and 56 urban health extension workers (76 HEWs) and 198 other health professionals working in the city with potential health service coverage of $100 \%$. Bahir Dar city has projected total population of 267,350 for $2012 / 13$.

\subsection{Study Design}

Unmatched community based case control and descriptive cross sectional investigation was conducted in sixty eight cases matched with one hundred thirty six controls that had no previous history of relapsing fever living in the same village as the cases.

Line list of cases was taken and followed daily within the study period, cases and controls interviewed using semi-structured questionnaire, their sleeping spaces were observed and all information hypothesized as risk factors for the relapsing fever outbreak was collected.

\subsection{Study Period}

Study was undertaken in all patients from 15 October to 15 November 2012.

\section{Results}

From a total of sixty-eight cases seen from 15 October to 15 November 2012 in the city, the overall attack rate (AR) was 0.26 per $1000(68)$ population (total population of Bahir-Dar). There was no death during the outbreak. Sixty two $(91.2 \%)$ of the cases were males and $66.2 \%$ (45) were within age range of 14-24 years, 30.9\% (21) were between $25-35$ years, and $1.5 \%$ (one) was $46+$ years of age. The age ranges from 12 to 56 years and $32.4 \%$ (22) of the cases were within 15-18 years of age. From total confirmed cases $100 \%$ (68), $92.6 \%$ ( 63), 94.1\% (64) and 82.3\% (56) presented with fever, head ache, chills and vomiting respectively.

The cases were those present themselves to Felegehiwot referral hospital, Bahir Dar health center, Han health center and Agmas private clinic. The median age was 20 years (SD 7.14) with range of 12 to 56 years. The majority $70.8 \%$ (48) were those between 12-24 years. Of the interviewed cases $91.2 \%$ (62) were daily laborers and only $8.8 \%$ (six) were children of those owners renting houses for daily laborers.

Bivariate logistic regression analysis of respondents of the study $75 \%$ (51) patients and $27.2 \%$ (37) controls did not wash their clothes at least weekly $(\mathrm{OR}=8.03,95 \% \mathrm{CI}$ [4.12-15.63] and p-value 0.000) and 79.4\% (54) patients and $64 \%(87)$ controls were not take body bath at least weakly $(\mathrm{OR}=2.17,95 \% \mathrm{CI}[1.09-4.31]$ and $\mathrm{p}$-value 0.037$)$ and the associations were statistically significant. The likelihood of acquiring relapsing fever for those not washing clothes at least weekly was about eight times and that of not taking body bath at least weekly was two times higher compared to those washing cloths and taking bath at least weekly(table 1).

Multivariate analysis of not wash clothes at least weekly $(\mathrm{AOR}=13.23,95 \%$ CI [5.51-31.75] $)$ and not take body bath at least weakly $(\mathrm{AOR}=8.01,95 \% \mathrm{CI}$ [3.51-18.29]) showed a statistically significant association. The likelihood of acquiring relapsing fever for those not washing clothes at least weekly was about thirteen times and that of not taking body bath at least weekly was eight times higher compared to washing cloths and taking bath at least weekly (table 1).

\subsection{Case-Control Study}

A total of 68 cases \& 136 controls were interviewed, of which sixty two $(91.2 \%)$ cases were males with the minimum age 12 and maximum 56 years old and mean age of respondents was 21 years and their median age was 20 years (SD 7.14).

Sixty one $(89.7 \%$ ) of the cases and $87.5 \%$ (119) controls were Orthodox in religion. $100 \%$ (204) cases and control 
were Amhara in ethnic, 39.7\% (27) cases and $33.1 \%(45)$ controls were illiterate, $51.5 \%$ (35) cases and $57.4 \%(78)$ controls were $1-4,2.9 \%(2)$ cases and $5.1 \%(7)$ controls were 5-8 grade and $5.9 \%(4)$ of the cases and $4.4 \%(6)$ of controls were 9-12 grade in education status.

Table 1. Bivariate and multivariate logistic regression analysis of factors for relapsing fever

\begin{tabular}{|c|c|c|c|c|c|}
\hline \multicolumn{2}{|c|}{ Variables } & \multirow{2}{*}{$\frac{\operatorname{Cases}(n=68)}{62(91.2 \%)}$} & \multirow{2}{*}{$\begin{array}{c}\text { Controls }(\mathbf{n}=\mathbf{1 3 6}) \\
125(92 \%)\end{array}$} & \multirow{2}{*}{$\begin{array}{c}\text { Crude OR, 95\% CI } \\
1\end{array}$} & \multirow[t]{2}{*}{ Adjusted OR, 95\% CI } \\
\hline & Male & & & & \\
\hline Sex & Female & $6(8.8 \%)$ & $11(8 \%)$ & $0.9,0.32-2.57$ & NI \\
\hline \multirow{4}{*}{ Age group in years } & $<15$ & $7(10.3 \%)$ & $12(8.8 \%)$ & $0.286,0.028-2.88$ & NI \\
\hline & $15-24$ & $41(60.3 \%)$ & $79(58.1 \%)$ & $0.321,0.037-2.758$ & NI \\
\hline & $25-24$ & $19(27.9 \%)$ & $39(28.7 \%)$ & $0.34,0.03-3.047$ & NI \\
\hline & $\geq 35$ & $1(1.5 \%)$ & $6(4.4 \%)$ & 1 & \\
\hline \multirow{4}{*}{ Educational status } & Illiterates & $27(39.7 \%)$ & $45(33.1 \%)$ & $1.769, .468-6.687$ & NI \\
\hline & 1-4 grade & $35(51.5 \%)$ & $78(57.4 \%)$ & $2.229, .606-8.195$ & NI \\
\hline & $5-8$ grade & $2(2.9 \%)$ & $7(5.1 \%)$ & $3.5, .473-25.901$ & NI \\
\hline & $\geq 9$ grade & $4(5.9 \%)$ & $6(4.4 \%)$ & 1 & \\
\hline \multirow{2}{*}{$\begin{array}{l}\text { Taking bath at least } \\
\text { weekly }\end{array}$} & No & $54(79.4 \%)$ & $87(64 \%)$ & $2.17,1.095-4.31 *$ & $8.01,3.51-18.29$ \\
\hline & Yes & $14(20.6 \%)$ & $49(36 \%)$ & 1 & 1 \\
\hline \multirow{2}{*}{$\begin{array}{l}\text { Washing clothes at } \\
\text { least weekly }\end{array}$} & No & $51(75 \%)$ & $37(27.2 \%)$ & $8.03,4.12-15.63 *$ & $13.23,5.51-31.75$ \\
\hline & Yes & $17(25 \%)$ & $99(72.8 \%)$ & 1 & 1 \\
\hline
\end{tabular}

* Significant at $95 \%$ CI

NI-not included

No statistical difference was found on age, sex, ethnicity and educational status compared both cases and control.

Thirteen (19.1\%) of the cases and $27.2 \%$ ( 37) controls had soap during interview time and $45.6 \%$ (31), $27.9 \%$ ( 19), $19.1 \%$ ( 13) and $7.4 \%$ ( 5) stayed for 2,3,4,5 days before seeking treatment respectively.

There was no shortage of water for personal hygiene in both cases and controls. Hundred percent of patients treated with antibiotics and Intra Venous fluid. All cases completely recovered from their illness.

Sixty eight (100\%) of the cases had fever, $82.3 \%(56)$ of cases had vomiting and $92.6 \%$ (63) had head ache, $94.1 \%$ (64) had chills and $62.6 \%(29)$ had jaundice.

\subsection{Observation of the situation of daily laborers}

- $\quad$ The minimum number of daily laborers who slept in a small room was five and the maximum was 80

- Almost all daily laborers had no alternative cloth for day and night

- No shortage of water supply for personal hygiene, drinking and sanitation purpose for all daily laborers.

\subsection{Actions Taken}

- Discussion was made with regional health bureau public health emergency coordinator and city health department public health emergency officer and the team engaged in activating epidemic response task force to participate in active case detection \& educating at gatherings to prevent and control the outbreak.

- The team also conducted daily reporting cases to next level

- Supportive supervision in the treatment sites.

- Delousing of cloths and shaving of hair of cases

- Mass sleeping houses (363) sprayed with malathion within six days (29 October-3 November 2012)(figure $1)$.

\section{Discussion}

The onset date of the first case was on $22^{\text {nd }}$ of September 2012 in Bahir Dar reported by the city administration health office. But the study was conducted from 15 October- 15 November 2012(figure1). Among all cases 91.2\% (62) were males and $66.2 \%$ (45) were within age range of $14-24$ years, $30.9 \%$ (21) were between $25-35$ years, and $1.5 \%$ (one)was $46+$ years of age. $32.4 \%$ (22) of the cases were within 15-18 years of age. The age ranges from 12 to 56 years. 


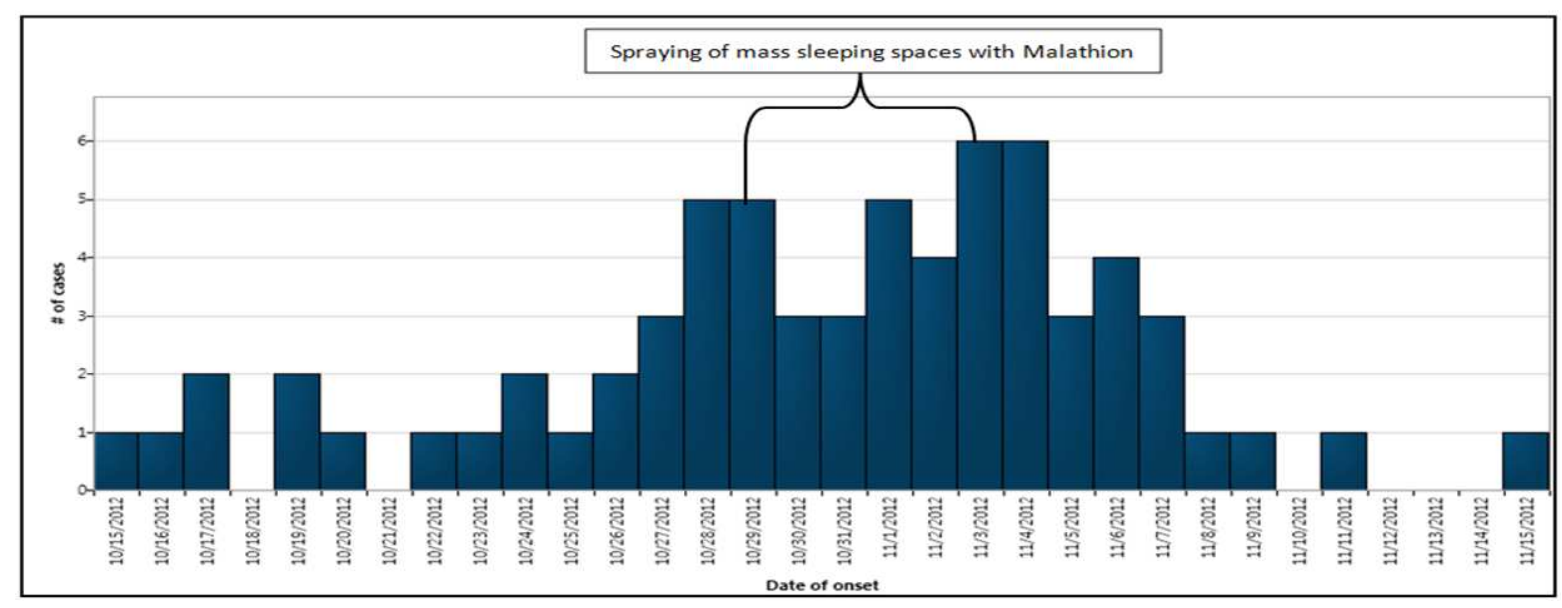

Figure 1. Epi curve of relapsing fever in Bahir-Dar, Amhara region, from 15 October-15 November 2012

The reason for rapid spread of outbreak might be due to poor personal hygiene, overcrowding and lack of alternative clothes. The number of cases was three times more than the previous year (2011) cases (only 22cases) reported to the regional health bureau in the same period. The evidence showed that all daily laborers who live in poor personal hygiene and overcrowding condition were at risk of the outbreak.

The overall attack rate (AR) was 0.26 per 1000 populations and there was no death during the outbreak as compared with outbreaks occurred in other parts of Ethiopia. This could be due to early detection and treatment and good case management in the treatment sites; $(1.9 \%$ in Asella hospital, 3.6\% in Hosaena hospital and 4.6\% Gondar, Ethiopia). Highest number of cases arose from Gish Abay $30.9 \%$ (21), Sefene-Selam 27.7\% (19) and Belay Zeleke $20.6 \%$ (14)kebeles may be due to the presence of a number (around 206) of mass sleeping houses for renting with low cost (three birr per day per head) in these kebeles and most of the time these kebeles were centers of daily labourers. After discussion, action plan was designed with regional, City administration and health facility authorities on prevention and control activities.

\section{Conclusion}

The attack rate (AR) was 0.26 per 1000 populations and there was no death. The number of cases $66.2 \%$ (45) were within age range of $14-24$ years, $30.9 \%$ (21) were between 25-35 years, and $1.5 \%$ (one) was $46+$ years of age. Poor personal hygiene among daily laborers, overcrowding (close contact) and lack of alternative clothes might contribute to increase the magnitude of the outbreak.

Exchanging information between different levels and providing prompt response in reducing undesirable disease outcomes, increasing daily laborer's awareness, capacity building to health personnel, permanent solution to interrupt the occurrence and distribution of the relapsing fever out-break requires more effort from all government and stake-holders.
This finding suggests that relapsing fever was a threat to daily laborers.

To prevent subsequent relapsing fever outbreaks the following action points put as recommendation:

1. Organize zonal meeting session with representative from all sectors including the zonal administrators to discuss the way how to minimize mass sleeping houses and solutions for daily laborers

2. Increase daily laborer's awareness through continuous health information

3. Daily laborers must wash cloths and take bath at least weekly

4. Close supportive supervision by city health department

\section{References}

[1] Schwan TG, Burgdorfer W, Rosa PA, Borrelia. Washington DC: ASM Press, 1995: 626-35

[2] Kasper B. Fauci, Hauser L. Jamesa. Harrison's principles of internal medicine: 16th edition, McGraw-Hill medical publishing division; 2005.

[3] Epidemiology and Ecology of Health and Disease in Ethiopia.

[4] Worku L., Solomon G. Louse-borne relapsing fever profile.

[5] Rebaudet S, Parola P. Epidemiology of relapsing fever and Borreliosis in Europe. FEMS Immunology and Med Microbiology. 2006 Oct; 48(1):11-5.

[6] David L.Heymann. Control of Communicable Diseases manual.

[7] Characteristics of louse-borne relapsing fever in Ethiopian children and adults.

[8] Jose M. Ramos, Eduaro M., Francisco R., Abraham T. Louse borne relapsing fever in Ethiopian children: Experience of a rural hospital.

[9] G Barbour, MD Microbiology, pathogenesis, and epidemiology of relapsing fever. 